\title{
Azimuthal magnetorotational instability with super-rotation
}

\author{
G. Rüdiger ${ }^{1} \dagger$, M. Schultz ${ }^{1}$, M. Gellert ${ }^{1}$ and F. Stefani $^{2}$ \\ ${ }^{1}$ Leibniz-Institut für Astrophysik Potsdam, An der Sternwarte 16, D-14482 Potsdam, Germany \\ ${ }^{2}$ Helmholtz-Zentrum Dresden-Rossendorf, PF 510119, 01314 Dresden, Germany
}

(Received xx; revised xx; accepted xx)

It is demonstrated that the azimuthal magnetorotational instability (AMRI) also works with radially increasing rotation rates contrary to the standard magnetorotational instability for axial fields which requires negative shear. The stability against nonaxisymmetric perturbations of a conducting Taylor-Couette flow with positive shear under the influence of a toroidal magnetic field is considered if the background field between the cylinders is current-free. For small magnetic Prandtl number Pm $\rightarrow 0$ the curves of neutral stability converge in the Hartmann number/Reynolds number plane approximating the stability curve obtained in the inductionless limit $\mathrm{Pm}=0$. The numerical solutions for $\mathrm{Pm}=0$ indicate the existence of a lower limit of the shear rate. For large Pm the curves scale with the magnetic Reynolds number of the outer cylinder but the flow is always stable for magnetic Prandtl number unity as is typical for doublediffusive instabilities.

We are particularly interested to know the minimum Hartmann number for neutral stability. For models with resting or almost resting inner cylinder and with perfect-conducting cylinder material the minimum Hartmann number occurs for a radius ratio of $r_{\mathrm{in}}=0.9$. The corresponding critical Reynolds numbers are smaller than $10^{4}$.

Key words: Astrophysical plasma - Plasma instabilities

\section{Introduction}

In recent years instabilities in rotating conducting fluids under the influence of magnetic fields became of high interest. Especially in view of astrophysical applications the consideration of differential rotation $\Omega=\Omega(R)$ is relevant. It has been known for a long time that differential rotation with negative shear $(\mathrm{d} \Omega / \mathrm{d} R<0$, 'sub-rotation') becomes unstable under the influence of an axial field (Velikhov 1959; Balbus \& Hawley 1991). When considered separately, both ingredients of this MagnetoRotational Instability (MRI), i.e. the axial field and also the Kepler rotation, are stable. The full system proves to be unstable if and only if the fluid is sub-rotating. For ideal fluids the criterion of stability against axisymmetric perturbations of differential rotation under the presence of toroidal fields $B_{\phi}$ reads

$$
\frac{1}{R^{3}} \frac{\mathrm{d}}{\mathrm{d} R}\left(R^{2} \Omega\right)^{2}-\frac{R}{\mu_{0} \rho} \frac{\mathrm{d}}{\mathrm{d} R}\left(\frac{B_{\phi}}{R}\right)^{2}>0,
$$

where $\mu_{0}$ is the magnetic permeability, $\rho$ the mass density and $R$ the distance from the rotation axis (Michael 1954).

The stability problem of toroidal fields plus differential rotation in cylindric geometry has been studied by several authors often considering the fluid as ideal and/or the perturbations as axisymmetric (Chandrasekhar 1961; Gotoh 1962; Howard \& Gupta 1962; Chanmugam 1979; Fearn

$\dagger$ Email address for correspondence: gruediger@aip.de 
1984; Balbus \& Hawlev 1992; Knobloch 1992; Dubrulle \& Knobloch 1993; Ogilvie \& Pringle 1996; Terquem \& Papaloizou 1996; Pessah \& Psaltis 2005; Shalybkov 2006). In view of possible experiments in the present paper the perturbations are considered as nonaxisymmetric existing in a diffusive fluid under the influence of a current-free toroidal magnetic field.

After (1.1) solid-body rotation and rotation laws with positive shear $(\mathrm{d} \Omega / \mathrm{d} R>0$, 'superrotation') are stabilized by the magnetic field unless the field strongly increases outwards. The profiles $B_{\phi} \propto 1 / R$ (current-free between the cylinders) and $B_{\phi} \propto R$ ( $z$-pinch, homogeneous axial electric-current) cannot destabilize rotation increasing radially if only axisymmetric perturbations are considered. It has even been demonstrated that for fields with $B_{\phi} \propto 1 / R$ all Taylor-Couette flows are stable against axisymmetric perturbations (Herron \& Soliman 2006). The Azimuthal MagnetoRotational Instability (AMRI) of differential rotation plus azimuthal fields current-free between the cylinders is thus basically nonaxisymmetric. Standard MRI and AMRI have in common that in both cases the magnetic background fields are force-free and that the combination of two stable components leads to instability. If the azimuthal magnetic field is not current-free and axial electric background currents exist we speak about Tayler instability (TI), which even exists without any rotation.

Taylor-Couette flows with positive shear are the prototype of hydrodynamic stability for moderate Reynolds numbers (Wendt 1933; Schultz-Grunow 1959). For finite, but very large, Reynolds numbers the existence of a linear instability for super-rotating Taylor-Couette flow has been reported recently (Deguchi 2017). All the more surprising is the finding that weak and stable magnetic azimuthal fields can destabilize also flows with radially increasing $\Omega$ of rather low Reynolds number if nonaxisymmetric perturbations are taken into account (Stefani \& Kirillov 2015; Rüdiger et al. 2016). Even in this particular combination of two highly stable ingredients the resulting MHD flow becomes unstable. We shall also demonstrate that the radial profile of the fields - if not too steep - does not play an important role for the question of stability or instability. On the other hand, the new instability is a double-diffusive phenomenon as it does not appear if the molecular viscosity and the molecular magnetic resistivity are equal. Contrary to AMRI, the standard MRI with axial magnetic fields and differential rotation with negative shear has no counterpart for positive shear.

The equations of the problem are

$$
\begin{aligned}
\frac{\partial \boldsymbol{U}}{\partial t}+(\boldsymbol{U} \cdot \nabla) \boldsymbol{U} & =-\frac{1}{\rho} \nabla P+\nu \Delta \boldsymbol{U}+\frac{1}{\mu_{0} \rho} \operatorname{curl} \boldsymbol{B} \times \boldsymbol{B}, \\
\frac{\partial \boldsymbol{B}}{\partial t} & =\operatorname{curl}(\boldsymbol{U} \times \boldsymbol{B})+\eta \Delta \boldsymbol{B}
\end{aligned}
$$

with $\operatorname{div} \boldsymbol{U}=\operatorname{div} \boldsymbol{B}=0$ for an incompressible fluid. $\boldsymbol{U}$ is the velocity, $\boldsymbol{B}$ the magnetic field vector, $P$ the pressure, $\nu$ the kinematic viscosity and $\eta$ is the magnetic resistivity. The basic state in the cylindric system with the coordinates $(R, \phi, z)$ is $U_{R}=U_{z}=B_{R}=B_{z}=0$ for the poloidal components and $\Omega=a_{\Omega}+b_{\Omega} / R^{2}$ for the rotation law with the constants

$$
a_{\Omega}=\frac{\mu-r_{\mathrm{in}}^{2}}{1-r_{\mathrm{in}}^{2}} \Omega_{\mathrm{in}}, \quad b_{\Omega}=\frac{1-\mu}{1-r_{\mathrm{in}}^{2}} \Omega_{\mathrm{in}} R_{\mathrm{in}}^{2} .
$$

Here $r_{\text {in }}=R_{\text {in }} / R_{\text {out }}$ is the ratio of the inner cylinder radius $R_{\text {in }}$ and the outer cylinder radius $R_{\text {out }} . \Omega_{\text {in }}$ and $\Omega_{\text {out }}$ are the angular velocities of the inner and outer cylinders, respectively. With the definition

$$
\mu=\frac{\Omega_{\mathrm{out}}}{\Omega_{\mathrm{in}}}
$$

super-rotation is represented by $\mu>1$. 
For the magnetic field the stationary solution is

$$
B_{\phi}=a_{B} R+\frac{b_{B}}{R} .
$$

The radial magnetic profile $B_{\phi} \propto R$ is due to an applied homogeneous axial electric current while $B_{\phi} \propto 1 / R$ is current-free in the fluid. We define $\mu_{B}=B_{\text {out }} / B_{\text {in. }}$. The current-free field is then given by $\mu_{B}=r_{\mathrm{in}}$ and the field in the $z$-pinch is $\mu_{B}=1 / r_{\mathrm{in}}$. Almost always in the present paper a narrow gap with $r_{\text {in }}=0.9$ is considered.

The dimensionless physical parameters of the system are the magnetic Prandtl number Pm, the Hartmann number Ha and the Reynolds number Re, i.e.

$$
\mathrm{Pm}=\frac{\nu}{\eta}, \quad \mathrm{Ha}=\frac{B_{\text {in }} D}{\sqrt{\mu_{0} \rho \nu \eta}}, \quad \mathrm{Re}=\frac{\Omega_{\text {out }} D^{2}}{\nu} .
$$

The magnetic Reynolds number is $\mathrm{Rm}=\mathrm{Pm} \cdot \mathrm{Re}$. The parameters combine to the magnetic Mach number

$$
\mathrm{Mm}=\frac{R_{\text {out }}}{D} \frac{\sqrt{\mathrm{Pm}} \mathrm{Re}}{\mathrm{Ha}},
$$

indicating whether the rotation energy dominates the magnetic energy or not. The parameter $D=R_{\text {out }}-R_{\text {in }}$ is the gap width. The Hartmann number is defined by the magnetic field on the inner wall where it is maximal. If the Reynolds number is defined with the rotation rate of the outer cylinder, then $\operatorname{Re} / \mu$ describes the Reynolds number of the inner cylinder. $\mu=\infty$ gives a model where the inner cylinder rests.

The variables $\boldsymbol{U}, \boldsymbol{B}$ and $P$ are split into mean and fluctuating components, i.e. $\boldsymbol{U}=\overline{\boldsymbol{U}}+$ $\boldsymbol{u}, \boldsymbol{B}=\overline{\boldsymbol{B}}+\boldsymbol{b}$ and $P=\bar{P}+p$. The bars from the variables are immediately dropped, so that the upper-case letters $\boldsymbol{U}, \boldsymbol{B}$ and $P$ represent the background quantities. By developing the disturbances $\boldsymbol{u}, p$ and $\boldsymbol{b}$ into normal modes the solutions of the linearized MHD equations

$$
[\boldsymbol{u}, \boldsymbol{b}, p]=[\boldsymbol{u}(R), \boldsymbol{b}(R), p(R)] \mathrm{e}^{\mathrm{i}(\omega t+k z+m \phi)},
$$

are considered for axially unbounded cylinders. Here $k$ is the axial wave number of the perturbation, $m$ its azimuthal wave number and $\omega$ the complex frequency including growth rate as its imaginary part and a drift frequency $\omega_{\mathrm{dr}}$ as its real part. A linear code is used to solve the resulting set of linearized ordinary differential equations for the radial functions of flow, field and pressure fluctuations. The solutions are optimized with respect to the Reynolds number for given Hartmann number by varying the wave number. Only solutions for $m=1$ are here discussed. The hydrodynamic boundary conditions at the cylinder walls are the rigid ones, i.e. $u_{R}=u_{\phi}=u_{z}=0$. The cylinders are assumed to be perfectly conducting or - in a few cases insulating. For the conducting walls the fluctuations $\boldsymbol{b}$ must fulfill $\mathrm{d} b_{\phi} / \mathrm{d} R+b_{\phi} / R=b_{R}=0$ at $R_{\text {in }}$ and $R_{\text {out }}$ so that ten boundary conditions exist for the set of ten differential equations. For $B_{z}=0$ one can easily show that the system is degenerate under the transformation $m \rightarrow-m$ so that all eigenvalues (for specific Re and $\mathrm{Ha}$ ) are valid for each pair $m= \pm 1$.

\section{Azimuthal magnetorotational instability}

Consider rotation laws where the outer cylinder rotates with higher frequency than the inner cylinder. For the small magnetic Prandtl number of $\mathrm{Pm}=10^{-5}$ Fig. 1 presents the lines of marginal instability (i.e. for vanishing growth rate) for the two rotation profiles with $\mu=1.9$ and $\mu=2.3$ for a narrow gap with $r_{\text {in }}=0.9$. The magnetic field between the perfect-conducting cylinders is fixed to the vacuum-type, $B_{\phi} \propto 1 / R$. The form of the resulting neutral lines corresponds to the well-known form for sub-rotation as a tilted cone with both branches having 


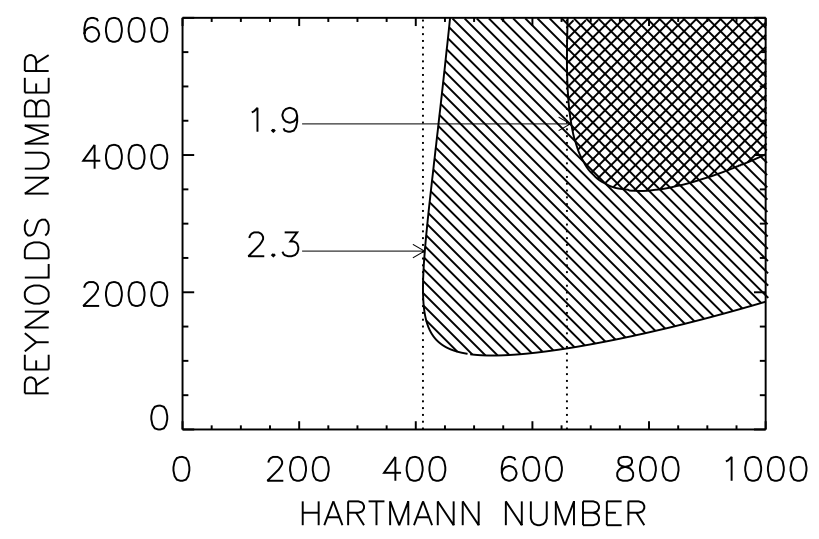

FIGURE 1. Instability cones for two rotation profiles with $\mu=1.9$ and $\mu=2.3$. By the vertical dotted lines the minimum Hartmann numbers $\mathrm{Ha}_{\min }$ are defined. $\mu_{B}=r_{\text {in }}=0.9, m= \pm 1, \mathrm{Pm}=10^{-5}$. The stability lines are also valid for $\mathrm{Pm}=0$. Perfect-conducting cylinders.
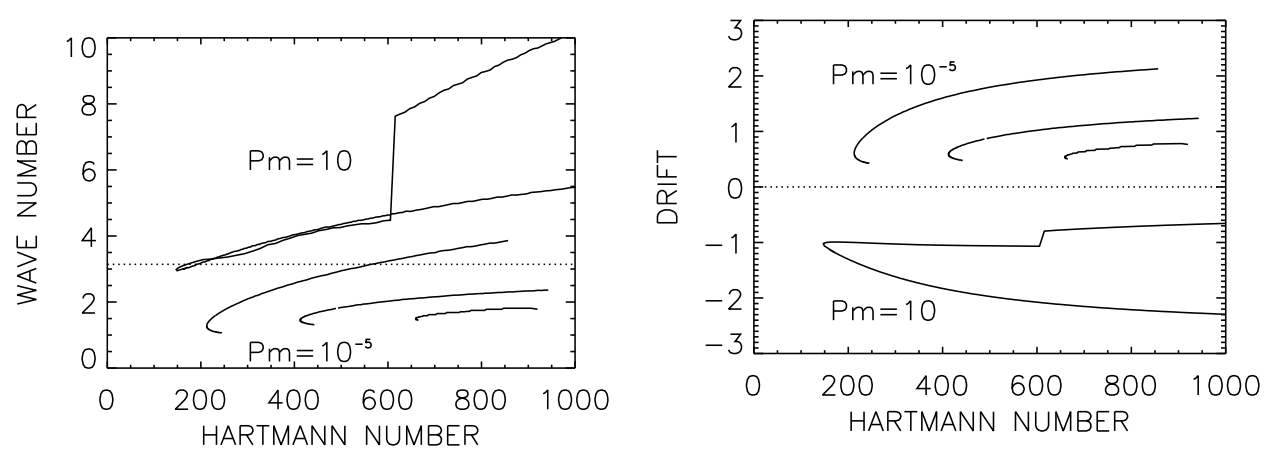

FIGURE 2. The axial wave number $k D$ (left panel) and the drift frequency $\omega_{\mathrm{dr}} / \Omega_{\text {out }}$ (right panel) along the lines of marginal instability for $\mathrm{Pm}=10^{-5}(\mu=5,2.3,1.9$, from left to right $)$ and $\mathrm{Pm}=10(\mu=5)$. $\mu_{B}=r_{\text {in }}=0.9, m= \pm 1$. Perfect-conducting cylinders.

a positive slope (Hollerbach et al. 2010; Rüdiger et al. 2013). Absolute minima of the Hartmann number and the Reynolds number exist, below which the rotation law is stable. Above the minima the instability domain is always limited by two critical values of the Hartmann number or the Reynolds number. For a given supercritical Reynolds number there is a minimum magnetic field for the instability and there is a maximum magnetic field destructing the instability. Also the Reynolds number can be too small or too large for a given Hartmann number. Above the upper branch of the instability curve the rotational shear is too strong to support nonaxisymmetric perturbations. Here we are in particular interested in the values of the absolute minimum $\mathrm{Ha}_{\text {min }}$ of the Hartmann number for neutral stability in order to discuss the possibility of laboratory experiments. Without magnetic field the flow is stable while the vertical dotted lines in Fig. 1 characterize the minimum magnetic field via $\mathrm{Ha}_{\text {min }}$.

The left panel of Fig. 2 gives the optimized axial wave numbers of the instability normalized with the gap width $D$ along the two branches of instability for small and large Pm. The axial cell size is $\delta z \simeq \pi D / k$. Cells with $k<\pi$ are thus prolate while cells with $k>\pi$ are oblate with respect to the rotation axis. The limit $k=\pi$ for nearly circular cells is marked by a horizontal dotted line. The plot demonstrates that the cell geometry strongly depends on the magnetic 

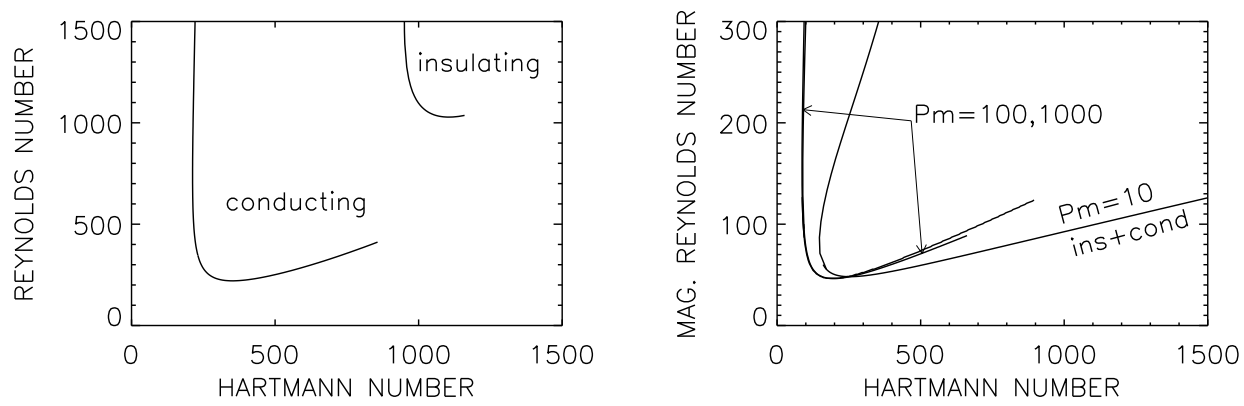

FIGURE 3. Stability maps for $\mu=5$ for perfect-conducting cylinders for $\mathrm{Pm}=10^{-5}$ (left) and $\mathrm{Pm} \gg 1$ (right). The lines for $\mathrm{Pm}=100$ and $\mathrm{Pm}=1000$ are almost indistinguishable. The left panel also contains the line of neutral stability for insulating cylinders; the curve for $\mathrm{Pm}=10$ (right panel) holds for insulating and perfect-conducting boundary conditions. Solutions for $\mathrm{Pm}=1$ do not exist. $\mathrm{Mm}<1$ for all curves. $\mu_{B}=r_{\text {in }}=0.9$.

Prandtl number. For small Pm also the wave numbers are small hence the cells are prolate or circular in the $(R, z)$ plane. Along the strong-field (lower) branch of the instability cone the axial wave numbers exceed those of the low-field (upper) branch where the cells are rather long in axial direction. For $\mathrm{Pm} \gg 1$, however, the wave numbers at both branches of the cone are larger than $\pi$ so that the cells are always very flat.

Also the drift rates possess a strong Pm-dependence. The drift values as given in the right panel of Fig. 2 are the real parts $\omega_{\mathrm{dr}}$ of the frequency $\omega$ of the Fourier mode of the instability normalized with the rotation rate of the outer cylinder. Because of

$$
\frac{\dot{\phi}}{\Omega_{\mathrm{out}}}=-\frac{\omega_{\mathrm{dr}}}{m \Omega_{\mathrm{out}}}
$$

the azimuthal migration $\dot{\phi}$ has the opposite sign of $\omega_{\mathrm{dr}}$. For AMRI with negative shear we always found that the pattern migrates for all Pm in positive $\phi$-direction (Rüdiger et al. 2014). For Tayler instability under the influence of radially increasing rotation the situation is more complicated as the pattern counter-rotates for small Pm while it co-rotates for Pm $>1$. Figure 2 shows similar results. For small $\mathrm{Pm}$ positive $\omega_{\mathrm{dr}}$ occur and the perturbation pattern indeed rotates retrograde. Large Pm provide negative drift values hence the pattern migrates in the direction of the rotation. For $\omega_{\mathrm{dr}} / \Omega_{\text {out }}=-1$ the pattern rotates just as the outer cylinder.

To see the influence of the boundary condition the left panel of Fig. 3 gives the stability map for $\mu=5$ for the two cases with perfect-conducting walls and insulating boundary conditions. The small magnetic Prandtl number of $\mathrm{Pm}=10^{-5}$ characterizes liquid sodium. For vacuum boundaries the rotation laws are much more stable than for perfect-conducting conditions. Note that $\mathrm{Ha}_{\text {min }}$ for insulating cylinders exceed the values of $\mathrm{Ha}_{\text {min }}$ for perfect-conducting cylinders by a factor of 4 . Also for AMRI with negative shear the critical Hartmann numbers and the Reynolds numbers for vacuum conditions strictly lie above the values for perfect-conducting conditions. One finds from the Figs. 13 the expected trend with $\mu$ for the minimum Hartmann number. For perfect-conducting boundaries and $\mathrm{Pm}=10^{-5}$ it decreases from $\mathrm{Ha}_{\mathrm{min}} \simeq 700$ for the flat rotation law with $\mu=1.9$ to $\mathrm{Ha}_{\text {min }} \simeq 200$ for the steeper rotation law with $\mu=5$. Below we shall discuss whether the reduction of $\mathrm{Ha}_{\text {min }}$ by growing rotation ratio $\mu$ is continued or not for $\mu \rightarrow \infty$, i.e. for stationary inner cylinder.

The right panel of Fig. 3 demonstrates instability also for $\mathrm{Pm} \gg 1$. The stability curves for $\mathrm{Pm}=10, \mathrm{Pm}=100$ and $\mathrm{Pm}=1000$ are given for perfect-conducting boundary conditions in the $\mathrm{Ha} / \mathrm{Rm}$ plane with $\mathrm{Rm}$ as the magnetic Reynolds number. Note that in this representation 

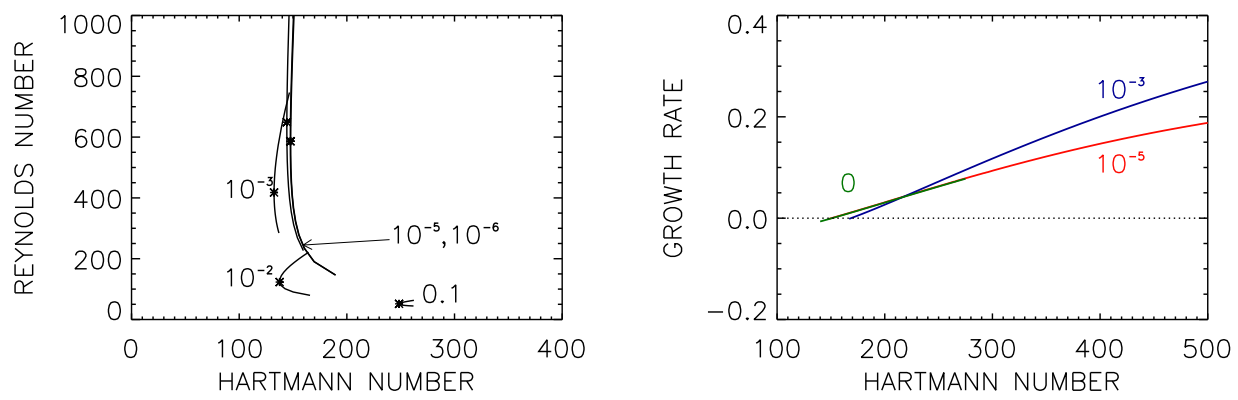

FIGURE 4. Left: Lines of neutral stability in the $(\mathrm{Ha} / \mathrm{Re})$ plane for various magnetic Prandtl numbers (marked) for almost stationary inner cylinder. For small Pm the lines coincide. Their minimum Hartmann number is $\mathrm{Ha}_{\min } \simeq 147$. Stars denote the $\mathrm{Ha}_{\min }$. Right: Growth rate normalized with $\Omega_{\text {out }}$ for $\mathrm{Re}=1000$ and three magnetic Prandtl numbers as indicated. $\mu_{B}=r_{\text {in }}=0.9, \mu=128$. Perfect-conducting cylinders.

the minimum values for Hartmann number and magnetic Reynolds number do hardly depend on $\mathrm{Pm}$. For $\mathrm{Pm} \rightarrow \infty$ the critical rotation rate appears to scale with $\mathrm{Rm}$. The curve for $\mathrm{Pm}=10$ simultaneously holds for the boundary conditions of both perfectly conducting and insulating cylinders. For $\mathrm{Pm}>1$ the large gaps between these curves known for $\mathrm{Pm}<1$ disappear. The role of the boundary conditions for the excitation of the instability strongly depends on the choice of the magnetic Prandtl number.

\section{Role of the magnetic Prandtl number}

We should also be interested to model containers with resting inner cylinder described by $\mu \rightarrow$ $\infty$ which our code approximates for very high $\mu$-values. One finds that already $\mu=128$ gives an excellent approximation of this rotation law. The left panel of Fig. 4 presents essential parts of the instability cones for $\mu=128$ for decreasing magnetic Prandtl numbers in the (Ha/Re) plane. For small Pm the curves perfectly coincide. The eigensolutions for neutral stability, therefore, scale with $\mathrm{Re}$ and $\mathrm{Ha}$ for $\mathrm{Pm} \rightarrow 0$. Hence, the numerical values of $\mathrm{Ha}$ and Re calculated with the inductionless approximation $\mathrm{Pm}=0$ can also be used for the small magnetic Prandtl numbers of gallium $\left(\mathrm{Pm} \simeq 10^{-6}\right)$ or even liquid sodium $\left(\mathrm{Pm} \simeq 10^{-5}\right)$. This approximation only models the relation $\nu \ll \eta$, it does not mean that $\nu=0$ (Roberts 1967). Below we shall demonstrate that the curves of neutral stability always scale with $\mathrm{Ha}$ and Re within this approximation . Note also how clear in Fig. 4 (left) the minimum Hartmann number $\mathrm{Ha}_{\min }$ stands at a constant value for the various small Pm.

For applications also the growth rates of the instability as the negative imaginary part of the Fourier frequency are relevant. They are normalized with the rotation rate $\Omega_{\text {out }}$ of the outer cylinder for a fixed Reynolds number and for almost-stationary inner cylinder by the right panel of Fig. 4 The common Reynolds number Re $=1000$ is the maximal value in the left panel. By definition the growth rates vanish for the marginal values of the Hartmann number. For $\omega_{\mathrm{gr}} / \Omega_{\mathrm{out}}=1$ the growth time relative to the rotation time (of the outer cylinder) is $\tau_{\mathrm{gr}} / \tau_{\text {rot }}=1 / 2 \pi$. We find for supercritical magnetic fields finite values of order 0.2 leading to the typical relation $\tau_{\mathrm{gr}} \simeq \tau_{\text {rot }}$. The Pm-dependence is only weak and disappears for $\mathrm{Pm} \rightarrow 0$. The lines converge for sufficiently small magnetic Prandtl number. Rotation with radially increasing frequency, therefore, magnetically decays and grows with the timescale of rotation itself - similar to the other forms of magnetorotational instability.

Figure 5 gives the minimum Hartmann number $\mathrm{Ha}_{\min }$ together with the associated Reynolds numbers for strong $(\mu=128)$ positive shear in dependence on $\mathrm{Pm}$. One finds convergency for 


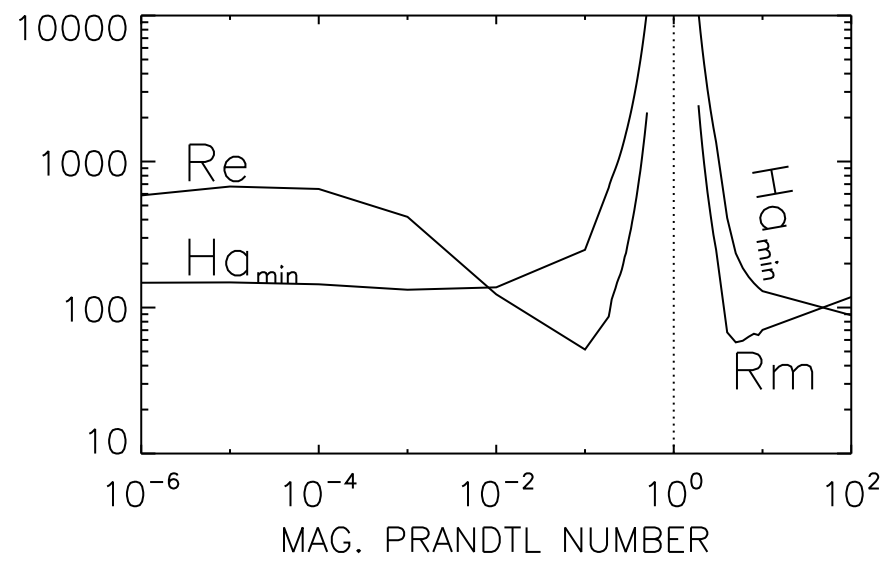

FIGURE 5. Minimum Hartmann number $\mathrm{Ha}_{\min }$ and the corresponding Reynolds numbers $(\mathrm{Pm}<1)$ and magnetic Reynolds numbers (Pm $>1$ ) in dependence on Pm for $\mu=128$. For Pm $=1$ solutions with positive growth rates do not exist. $\mu_{B}=r_{\text {in }}=0.9$. Perfect-conducting cylinders.

both eigenvalues for $\mathrm{Pm} \rightarrow 0$. The curves basically scale with $\mathrm{Ha}$ and $\mathrm{Re}$ for $\mathrm{Pm} \rightarrow 0$ in both cases.

For $\mathrm{Pm}<1$ the Reynolds number takes a minimum at $\mathrm{Pm} \simeq 0.1$. For even larger $\mathrm{Pm}$ it grows to infinity if $\mathrm{Pm} \rightarrow 1$. No solution for $\mathrm{Pm}=1$ exists. For even larger Pm, however, the instability is reanimated with Hartmann numbers of the same order as for $\mathrm{Pm}<1$ and rather low magnetic Reynolds numbers. Even for large magnetic Prandtl numbers the magnetic Mach number remains smaller than unity. Instabilities which only exist for $\nu \neq \eta$ belong to the class of double-diffusive instabilities (Acheson 1978). They do not appear for Pm $=1$ what possibly means that they do not exist in ideal fluids. Stefani \& Kirillov (2015) derived eigenvalues for rotation laws with positive shear and for $\mathrm{Pm}=0$ in a short-wave approximation without boundary conditions, demonstrating the local character of the instability.

In Fig. 5 the eigenvalues Re and $\mathrm{Ha}$ do not depend on $\mathrm{Pm}$ if $\mathrm{Pm} \rightarrow 0$. The question is whether the global equation system for the perturbations also possesses solutions for $\mathrm{Pm}=0$ for all $\mu>1$ or not. We already know that AMRI solutions with negative shear do exist for $\mathrm{Pm}=0$ but only for flows close to the Rayleigh line with $\mu \leqslant 0.3$ (Hollerbach et al. 2010). We ask whether such a limit also exists for super-rotation. The standard MRI (with axial fields) for all (negative) shear values does not exist for $\mathrm{Pm}=0$ (Chandrasekhar 1961). For Pm $\rightarrow 0$ it scales with the magnetic Reynolds number Rm leading for small Pm to very large Reynolds numbers. This is the explanation that the experimental realization of the standard MRI is still an existing challenge.

The dimensionless and linearized equations for the evolution of the flow, field and pressure perturbations in the inductionless (or 'quasistationary') approximation for $\mathrm{Pm}=0$ are

$$
\operatorname{Re}\left(\frac{\partial \boldsymbol{u}}{\partial \mathrm{t}}+(\boldsymbol{U} \cdot \nabla) \boldsymbol{u}+(\boldsymbol{u} \cdot \nabla) \boldsymbol{U}\right)=-\nabla p+\Delta \boldsymbol{u}+\mathrm{Ha}^{2}(\operatorname{curl} \boldsymbol{b} \times \boldsymbol{B}+\operatorname{curl} \boldsymbol{B} \times \boldsymbol{b})
$$

and

$$
\operatorname{curl}(\boldsymbol{u} \times \boldsymbol{B})+\Delta \boldsymbol{b}=0,
$$

together with $\operatorname{div} \boldsymbol{u}=\operatorname{div} \boldsymbol{b}=0$. The fluctuating and mean magnetic fields are normalized with a characteristic scale $B_{0}$ of the background field. The background flow $\boldsymbol{U}$ is normalized with 

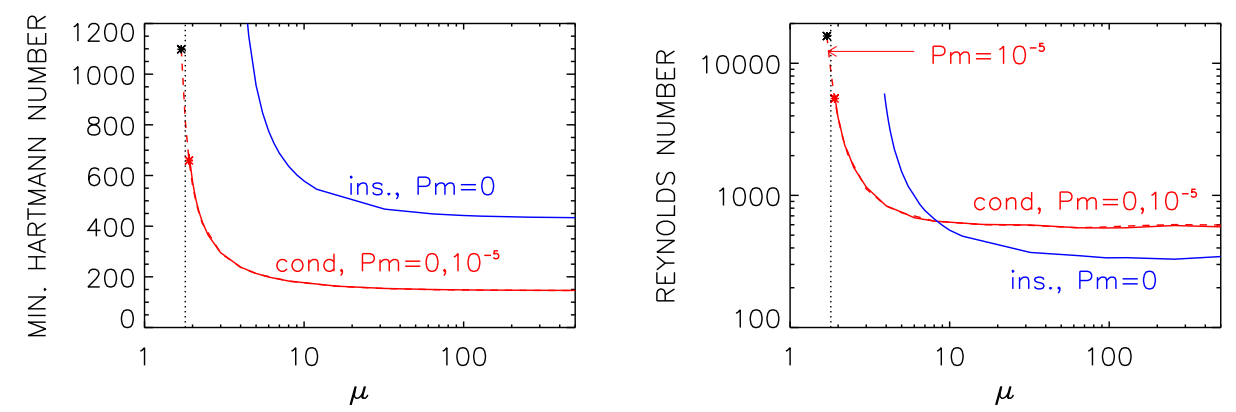

FIGURE 6. Hamin (left), the corresponding Reynolds numbers (right) versus the shear number $\mu$ for insulating cylinders (blue, $\mathrm{Pm}=0$ ) and perfect-conducting cylinders (red, $\mathrm{Pm}=0, \mathrm{Pm}=10^{-5}$ ). The vertical dotted lines mark shear of $\mu_{\text {crit }}=1.8$. For $\mathrm{Pm}=0$ no solution for $\mu<1.9$ (red dot) has been found. The dark dot represents the last known solution at $\mu=1.7$ for $\mathrm{Pm}=10^{-5}$. Note the crossing of the Reynolds number lines for $\mu \approx 8 . m= \pm 1, r_{\text {in }}=0.9$.

a characteristic flow amplitude $U_{0}$, the flow perturbations with $\eta / D$ and the time with $D / U_{0}$. Obviously, the eigenvalues of the system are Re and Ha.

The equation system (3.1) and (3.2) is numerically solved with the boundary conditions as described above. Figure 6 shows that for rotation with sufficiently large positive shear $\mu>$ $\mu_{\text {crit }}>1$ solutions for Pm $=0$ indeed exist for both sorts of boundary conditions. For perfectconducting boundaries the dotted vertical line at $\mu_{\text {crit }}=1.8$ appears as a lower bound for the eigensolutions. As indicated by black dots, for $\mathrm{Pm}=10^{-5}$ solutions also exist for $\mu<\mu_{\text {crit }}$ but only for very large Reynolds and Hartmann numbers. It is a similar situation as for negative shear where solutions for $\mu \gtrsim 0.3$ only exist for finite Pm with very large Reynolds numbers. Note that the lower bounds $\mu_{\text {crit }}$ are much harder to extrapolate for insulating boundary conditions than for perfect-conducting ones.

After Fig. 11 the global solution with $\mu=1.9$ and for $\mathrm{Pm}=10^{-5}$ requires much higher Hartmann numbers and Reynolds numbers than the steeper rotation law with $\mu=2.3$. Formally, the critical values for $\mu=1$ (rigid rotation) are infinite. It is insofar not surprising that both Reynolds numbers and Hartmann numbers are growing for $\mu \rightarrow 1$. For $\mathrm{Pm}=0$, however, no solution for $\mu<1.9$ has been found. The curves for $\mathrm{Pm}=0$ indeed suggest the existence of the lower limit $\mu_{\text {crit }}>1$ where the eigenvalues become infinite. Obviously, finite values of the magnetic resistivity stabilize too flat rotation laws with positive shear in the sense as Liu et al. (2006) found for the axisymmetric modes of helical magnetorotational instability within the inductionless short-wave approximation.

For conducting boundaries also the curves for $\mathrm{Pm}=10^{-5}$ in Fig. 6 are given showing nearly perfect coincidence with the curve for $\mathrm{Pm}=0$. The two lines hardly can be distinguished by eyes. The results of calculations in the inductionless approximation can thus be assumed as valid for liquid metals with their small Pm. Note also that for perfect-conducting cylinders much weaker magnetic fields than for insulating cylinders are needed for the instability onset. For large values of $\mu$ the ratio of the $\mathrm{Ha}_{\mathrm{min}}$ for insulating and perfect-conducting boundaries is about 2.5. The same is true with respect to the Reynolds number but only for small $\mu$ (flat rotation laws) while for large $\mu$ (steep rotation laws) the critical Reynolds numbers for vacuum conditions are smaller than for conducting boundary conditions. This behaviour of the eigenvalues is already known from standard MRI of quasi-Keplerian rotation laws with axial fields for different boundary conditions.

We ask for the azimuthal drift rates $\omega_{\mathrm{dr}} / \Omega_{\text {out }}$ of the solution with the lowest Hartmann number. In the right panel of Fig. 2 these values for $\mathrm{Pm}=10^{-5}$ only slightly vary with $\mu$ 


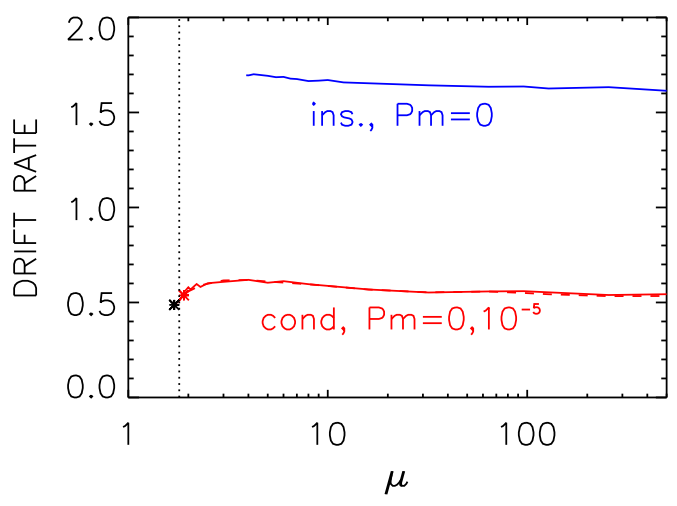

FIGURE 7. Same as in Fig. 6 but for the drift rate $\omega_{\mathrm{dr}} / \Omega_{\text {out }}$.

for $\mu \leqslant 5$. Figure 7 also shows the azimuthal drift measured in terms of the outer rotation rate hardly depending on the shear or the magnetic Prandtl number but its numerical value basically depends on the boundary condition. For both boundary conditions and for small Pm the instability pattern rotates retrograde. The amount of this migration basically depends on the material of the cylinders. The rotation rate of the inner cylinder does not at all influence the azimuthal migration of the pattern.

\section{Electric currents}

It remains to discuss the influence of the size of the gap between the cylinders on the excitation conditions and the axial electric current which produces the minimum Hartmann numbers.

A Taylor-Couette flow with resting inner cylinder is clearly the most simple model for superrotating fluids. As it also forms the steepest radial profile one expects for this configuration the lowest Hartmann numbers. From Fig. 6 we find, however, that both Reynolds and Hartmann numbers for $\mu \rightarrow \infty$ are well represented by the solutions already for $\mu=128$ and that perfectconducting boundaries always lead to the least numerical values. Figure 8 provides the results for variation of the gap width for $\mathrm{Pm}=0$ and for $0.7 \leqslant r_{\text {in }}<1$. The Hartmann number for perfectconducting boundaries has a minimum close to $r_{\mathrm{in}}=0.9$. In order to transform the values to the axial electric-current within the inner cylinder the relation $I_{\text {axis }}=5 R_{\text {in }} B_{\text {in }}$ (see Rüdiger et al. 2013) can be written as

$$
I_{\text {axis }}=5 \frac{R_{\text {in }}}{D} \mathrm{Ha} \sqrt{\mu_{0} \rho \nu \eta},
$$

where the axial currents are measured in Ampere. The numerical values are given as the thick dashed line in Fig. 8 . The result is that a minimum electric current is $26.1 \mathrm{kAmp}$ for liquid sodium as the conducting fluid 1 with a gap width of 0.25 .

The Reynolds numbers associated with the minimal Hartmann numbers given in Fig. 8 are moderate enough to ensure laminar flows in nonmagnetic experiments (see Balbus 2011). For stationary inner cylinders with $r_{\text {in }}=0.92$ Schultz-Grunow (1959) found stable solutions for Reynolds numbers not exceeding 40.000 in agreement with the data of Taylor (1936). Burin \& Czarnocki (2012) detailed discussed the phenomena of subcritical transition to turbulence in Taylor-Couette flows by finite disturbances. The experiment described by

$$
\dagger \sqrt{\mu_{0} \rho \nu \eta} \simeq 8.2 \text { in c.g.s. }
$$




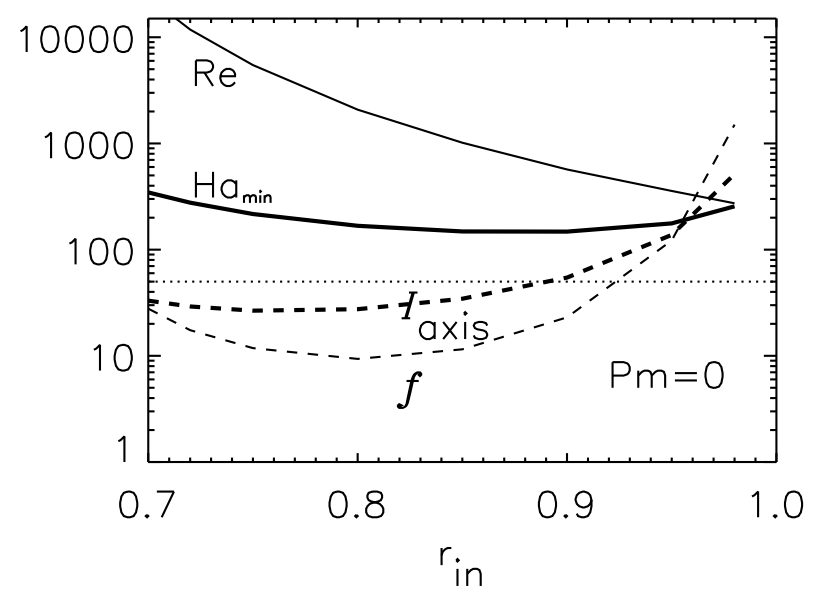

FIGURE 8. Hamin (thick solid line) and corresponding Reynolds number (thin solid line) versus $r_{\text {in }}$. The necessary axial electric current $I_{\text {axis }}$ (thick dashed line, in kAmp) and rotation frequency $f$ of the outer cylinder (thin dashed line, in $\mathrm{Hz}$ ) are also given for sodium as the conducting fluid. $R_{\text {out }}=5 \mathrm{~cm}, \mu=128$, $m= \pm 1, \mathrm{Pm}=0$. Perfect-conducting cylinders.
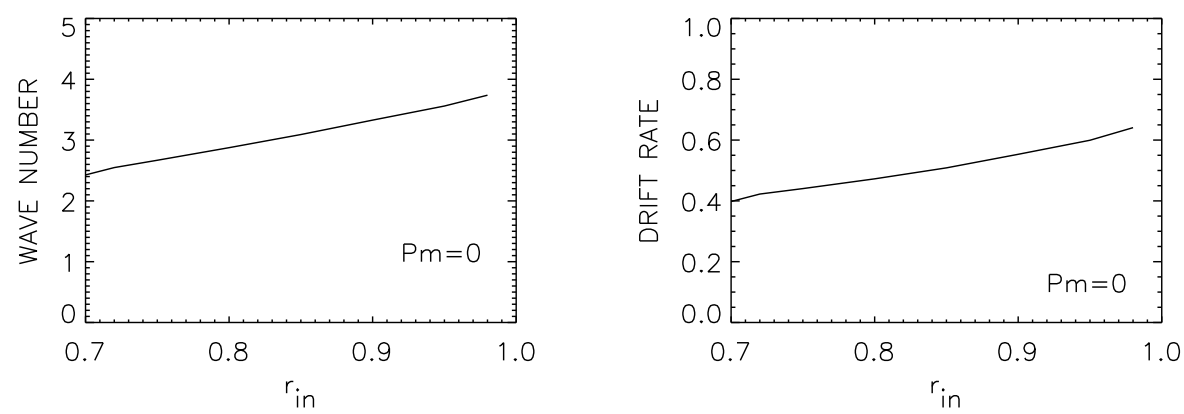

FIGURE 9. Same as in Fig. 8 but for the axial wave number $k D$ (left) and the azimuthal drift rate $\omega_{\mathrm{dr}} / \Omega_{\text {out }}$ (right).

Seilmayer et al. (2014) to realize the current-free AMRI for negative shear worked with $r_{\text {in }}=0.5$ (with outer radius $8 \mathrm{~cm}$ ) and a Reynolds number of 2956 without indication of any nonmagnetic instability within the container. Flows with positive shears are even more stable.

For increasing gap width the Reynolds number continuously grows. However, the rotation frequency $f=\Omega_{\text {out }} / 2 \pi$ possesses a minimum at $r_{\text {in }}=0.8$. For liquid sodium $(\nu=7.1$. $10^{-3} \mathrm{~cm}^{2} / \mathrm{s}$ ) and (say) $R_{\text {out }}=5 \mathrm{~cm}$ one obtains $f=9.3 \mathrm{~Hz}$. For wider gaps both the critical Reynolds number and Hartmann number rapidly increase. For $r_{\text {in }}=0.66$ we did not find any instability assuming $\operatorname{Re}<15.000$. Note that the size of the container does not influence the needed electric current for instability but the fractional gap width does.

The eigenvalues given in Fig. 8 are also characterized by associated axial wave numbers and azimuthal drift rates. The dependencies of these values on $r_{\text {in }}$ are not strong. For not too narrow gaps the cells are hardly elongated with the rotation axis and the azimuthal migration 2.1) of the instability pattern is always negative becoming (slightly) slower for wider gaps (Fig. 9). With the used normalizations both the axial wave number and the drift rates linearly grow with the value of $r_{\text {in }}$; they are maximal for $r_{\text {in }} \rightarrow 1$. 


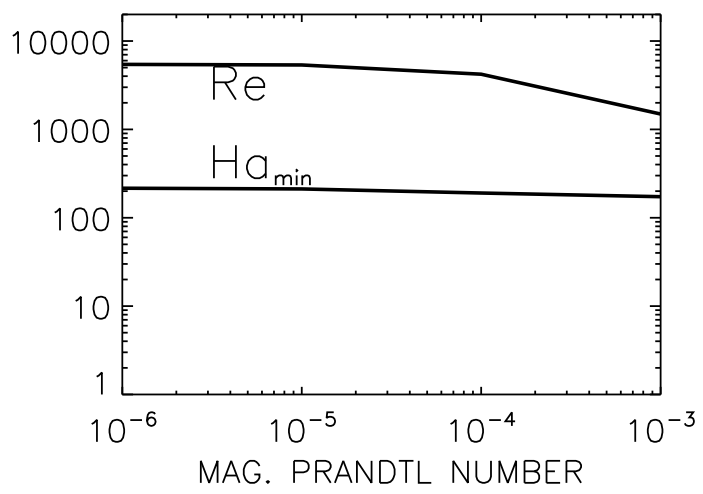

FIgURE 10. Same as in Fig. 5 but for $r_{\text {in }}=0.75$. For $\mathrm{Pm}=10^{-5}$ the Hartmann number is Hamin $=211.8$ and the Reynolds number is $\mathrm{Re}=5360$. Between $\mathrm{Pm} \simeq 10^{-3}$ and $\mathrm{Pm}=1$ the values rapidly grow to $\infty$. $\mu=128, \mu_{B}=r_{\text {in }}=0.75$. Perfect-conducting cylinders.

For the container with $r_{\text {in }}=0.75$, which after Fig. 8 requires the minimum axial electric current for excitation, the Fig. 10 again demonstrates the independence of Hartmann number and Reynolds number of the magnetic Prandtl number. Both values do not depend on Pm for $\mathrm{Pm} \lesssim 10^{-5}$. It is indeed allowed to use for the critical values $\mathrm{Ha}_{\min }$ and $\mathrm{Re}$ the results of the inductionless approximation for liquid metals such as mercury, gallium and sodium as the conducting fluids. The magnetic Mach number (1.7) for the largest Pm in Fig. 10 is of order unity but for smaller Pm - as for all instabilities which scale with $\mathrm{Ha}$ and $\mathrm{Re}$ for $\mathrm{Pm} \rightarrow 0$ - the magnetic Mach number is much smaller than unity.

After Eq. (4.1) the electric currents needed for neutral stability behave like the numerical values of the material expression $\sqrt{\mu_{0} \rho \nu \eta}$ which mainly runs with $\sqrt{\rho}$ as for the mentioned liquid metals the averaged diffusivity $\sqrt{\nu \eta} \approx$ const. It is thus clear that experiments for liquid sodium require the weakest electric currents.

Sofar we did not vary the radial profile of the magnetic background field. One of the questions is how sensitive the described instability reacts on the presence of axial electric-currents within the fluid. The Tayler instability of the $z$-pinch with $B_{\phi} \propto R$ - which for $\mathrm{Ha}=\mathrm{Ha}_{0}$ is unstable against nonaxisymmetric perturbations for all $\mathrm{Pm}$ even without rotation - is supported by super-rotation unless $\mathrm{Pm}=1$ so that even subcritical Hartmann numbers $\mathrm{Ha}<\mathrm{Ha}_{0}$ exist (Rüdiger et al. 2016). Similar to our above results the azimuthal migration of the nonaxisymmetric instability pattern depends on Pm. Also here the pattern counter-rotates for $\mathrm{Pm} \ll 1$ and it co-rotates for $\mathrm{Pm} \gg 1$.

In addition for the current-free field $B_{\phi} \propto 1 / R$ Fig. 11 also yields the stability lines for the field $B_{\phi} \propto R$ for two different magnetic Prandtl numbers. The instability for $\mathrm{Re}=0$ appears at $\mathrm{Ha}_{0}$ (Tayler 1957, 1973; Vandakurov 1972; Seilmayer et al. 2012) which does not depend on Pm (Rüdiger \& Schultz 2010). For large Reynolds numbers only small differences of the lines of marginal instability exist for the two radial profiles. The minimum Hartmann number for instability are slightly reduced by the electric currents, $\mathrm{Ha}_{\min }<\mathrm{Ha}_{0}$, but the differences are small. The main result is that the magnetic-induced instability of super-rotating Taylor-Couette flows with narrow gaps is only slightly influenced by the applied radial field profile. The upper branches in Fig. 11 demonstrate this phenomenon for two different magnetic Prandtl numbers. One can even consider the field as uniform between the cylinders (Edmonds 1958). The magnetic field only acts as a catalyst for the instability.

To visualize the shape of the instability pattern a nonlinear spectral code is used which has 


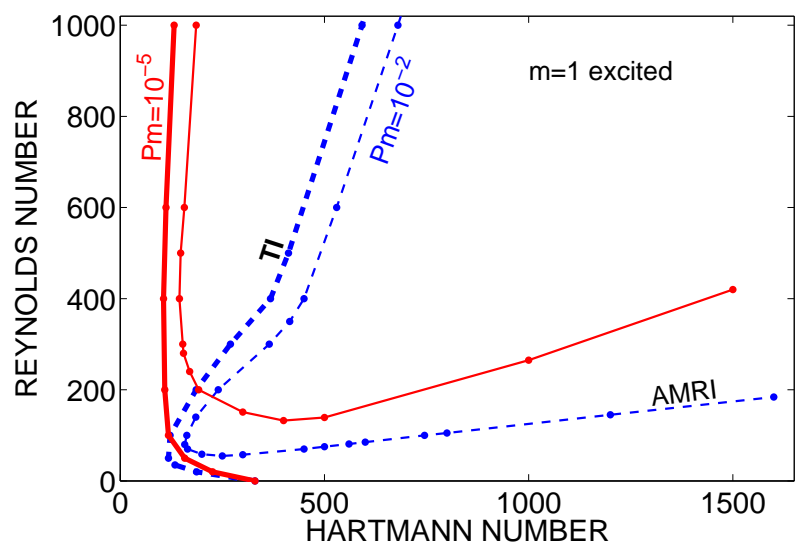

FIGURE 11 . Stability map for resting inner cylinder. The radial profiles of the background fields vary from $\mu_{B}=1 / r_{\text {in }}$ (TI, thick lines) to $\mu_{B}=r_{\text {in }}$ (AMRI, thin lines) and for $\mathrm{Pm}=0.02$ (blue) and $\mathrm{Pm}=10^{-5}$ (red). $\mathrm{Ha}_{0}=332$ for all Pm. Note the coincidence of the upper branches for large Reynolds numbers. $r_{\text {in }}=0.9$, perfect-conducting boundaries.
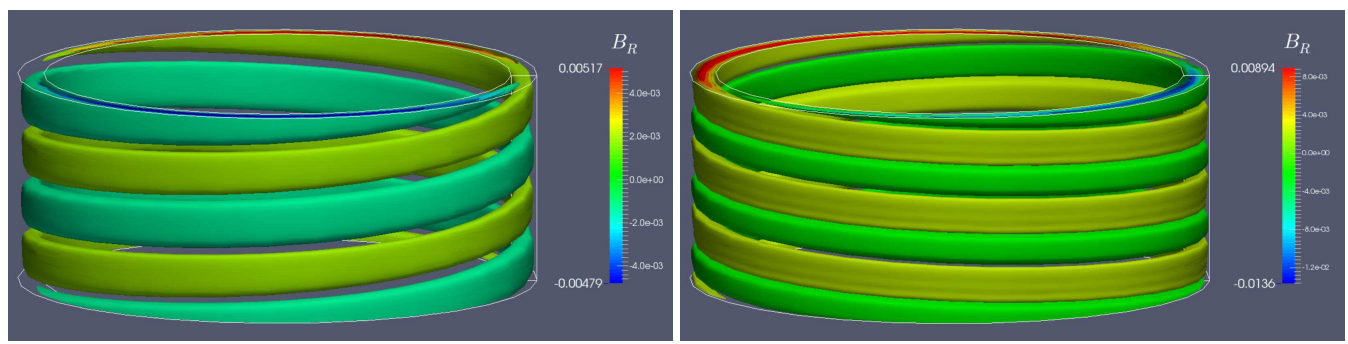

FIGURE 12. Isolines of the radial magnetic field component for instability without axial electric current between the cylinders $\left(\mu_{B}=r_{\text {in }}\right.$, left $)$ and with axial electric current $\left(\mu_{B}=1 / r_{\text {in }}\right.$, right). Resting inner cylinder. $\mathrm{Ha}=300, r_{\mathrm{in}}=0.9, \mathrm{Re}=155, \mathrm{Pm}=0.02$, perfect-conducting boundaries.

been developed from the hydrodynamic code of described by Fournier et al. (2004) and works with the expansion of the solution after azimuthal Fourier modes. Figure 12 demonstrates that the resulting patterns of the instabilities for resting inner cylinder and for $\mathrm{Pm}=0.02$ only sightly differ in dependence of the existence of axial electric background currents. The isolines of the radial magnetic field component are given showing different helicities of the two cases for the given sign of the electric current) and the axial wavelength in the left panel exceeds that of the instability model in the right panel.

The two examples in Fig. 12 have identical supercritical Reynolds number and Hartmann number. In the right panel a uniform axial electric current exists in the fluid between the cylinders so that the pattern belongs to the Tayler instability (under the influence of differential rotation). The electric current is missing in the left panel but the cell structure is very similar. For strong super-rotation the two instabilities appear to fuse and both instabilities are hard to distinguish unless $\mathrm{Pm}=1$.

\section{Conclusions}

In an earlier paper we have shown that Taylor-Couette flows with radially increasing rotation can become unstable against nonaxisymmetric perturbations under the presence of sufficiently strong azimuthal magnetic fields which are due to a homogeneous electric current (Rüdiger et al. 
2016). The present paper leads to the conclusion that such instability also exists if the field between the cylinders is current-free. At least for small Pm under the influence of differential rotation the differences between both instabilities become very small. Both instabilities appear for $\mathrm{Pm} \neq 1$ while the sign of the azimuthal pattern drift strongly differs for $\mathrm{Pm} \ll 1$ and $\mathrm{Pm} \gg 1$. For $\mathrm{Pm}=1$ rotation with positive shear stabilizes TI and completely suppresses the instability of current-free fields. Solutions for AMRI only exist if the molecular viscosity and the microscopic magnetic diffusivity strongly differ, hence solution do not exist for $\mathrm{Pm}=1$ which is the characteristics of double-diffusive instabilities.

An important difference to the standard magnetorotational instability with axial fields (which only exists for negative shear) is that the new instability also appears in the inductionless approximation with $\mathrm{Pm}=0$ for sufficiently large positive shear. The numerical calculations suggest a lower limit of $\mu_{\text {crit }}=1.8$. For finite but small magnetic Prandtl numbers solutions exist even below this limit but with very large Reynolds numbers. The consequence is that for finite but small magnetic Prandtl numbers the solutions for $\mu>\mu_{\text {crit }}$ scale with Re and Ha almost independent of $\mathrm{Pm}$. This gives the chance to probe the numerical results by experiments in the laboratory by use of liquid metals with their extremely low magnetic Prandtl numbers. For the optimal gap width of 0.25 and for liquid sodium as the conducting fluid the minimal axial current is $26.1 \mathrm{kAmp}$ and the rotation frequency is about $10 \mathrm{~Hz}$ (for $R_{\text {out }}=5 \mathrm{~cm}$ ). These numbers should be suitable for experiments. However, in view of the significant differences of $\mathrm{Ha}_{\text {min }}$ for perfect-conducting and insulating boundaries (Fig.6) any real experiment with sodium flowing between (say) copper walls remains to be carefully predicted.

\section{REFERENCES}

ACHESON, D. J. 1978 On the instability of toroidal magnetic fields and differential rotation in stars. Philosophical Transactions of the Royal Society of London Series A 289, 459-500.

BAlbus, S. A. 2011 Fluid dynamics: A turbulent matter. Nature 470, 475-476.

BAlbus, S. A. \& HAWLEY, J. F. 1991 A powerful local shear instability in weakly magnetized disks. I Linear analysis. II - Nonlinear evolution. The Astrophysical Journal 376, 214-233.

Balbus, S. A. \& Hawley, J. F. 1992 A Powerful Local Shear Instability in Weakly Magnetized Disks. IV. Nonaxisymmetric Perturbations. The Astrophysical Journal 400, 610-621.

Burin, M. J. \& CZARnOCKI, C. J. 2012 Subcritical transition and spiral turbulence in circular Couette flow. Journal of Fluid Mechanics 709, 106-122.

CHANDRASEKHAR, S. 1961 Hydrodynamic and hydromagnetic stability. Oxford: Clarendon.

Chanmugam, G. 1979 Adiabatic stability of toroidal magnetic fields in differentially rotating stars. Month. Not. Roy. Astr. Soc. 187, 769-776.

Deguchi, K. 2017 Linear instability in Rayleigh-stable Taylor-Couette flow. Physical Review E 95 (2), 021102.

Dubrulle, B. \& Knobloch, E. 1993 On instabilities in magnetized accretion disks. Astronomy \& Astrophysics 274, 667.

EdMonds, JR., F. N. 1958 Hydromagnetic Stability of a Conducting Fluid in a Circular Magnetic Field. Physics of Fluids 1, 30-41.

FEARN, D. R. 1984 Hydromagnetic waves in a differentialy rotating annulus. II - Resistive instabilities. Geophysical and Astrophysical Fluid Dynamics 30, 227-239.

Fournier, A., Bunge, H.-P., Hollerbach, R. \& Vilotte, J.-P. 2004 Application of the spectralelement method to the axisymmetric Navier-Stokes equation. Geophysical Journal International 156, 682-700.

Gotoh, K. 1962 Stability of a Flow between Two Rotating Cylinders in the Presence of a Circular Magnetic Field. Journal of the Physical Society of Japan 17, 1053-1061.

Herron, I. \& Soliman, F. 2006 The stability of couette flow in a toroidal magnetic field. Appl. Math. Lett. 19, 1113-1117.

Hollerbach, R., TeEluck, V. \& RÜDIger, G. 2010 Nonaxisymmetric Magnetorotational Instabilities in Cylindrical Taylor-Couette Flow. Physical Review Letters 104 (4), 044502. 
HowARD, L. N. \& GUPTA, A. S. 1962 On the hydrodynamic and hydromagnetic stability of swirling flows. Journal of Fluid Mechanics 14, 463-476.

KNobloch, E. 1992 On the stability of magnetized accretion discs. Month. Not. Roy. Astr. Soc. 255, 25P-28P.

LiU, W., Goodman, J., Herron, I. \& Ji, H. 2006 Helical magnetorotational instability in magnetized Taylor-Couette flow. Physical Review E 74 (5), 056302.

MichAEL, D. 1954 The stability of an incompressible electrically conducting fluid rotating about an axis when current flows parallel to the axis. Mathematika 1, 45-50.

Ogilvie, G. I. \& PRIngle, J. E. 1996 The non-axisymmetric instability of a cylindrical shear flow containing an azimuthal magnetic field. Month. Not. Roy. Astr. Soc. 279, 152-164.

Pessah, M. E. \& Psaltis, D. 2005 The Stability of Magnetized Rotating Plasmas with Superthermal Fields. The Astrophysical Journal 628, 879-901.

RoberTs, P. H. 1967 An Introduction to Magnetohydrodynamics. Longmans.

Rüdiger, G., Gellert, M., Schultz, M., Hollerbach, R. \& STEFani, F. 2014 Astrophysical and experimental implications from the magnetorotational instability of toroidal fields. Month. Not. Roy. Astr. Soc. 438, 271-277.

Rüdiger, G., Kitchatinov, L. L. \& Hollerbach, R. 2013 Magnetic Processes in Astrophysics: theory, simulations, experiments. Wiley-VCH.

RÜDIGER, G. \& SCHUltZ, M. 2010 Tayler instability of toroidal magnetic fields in MHD Taylor-Couette flows. Astronomische Nachrichten 331, 121.

Rüdiger, G., Schultz, M., Gellert, M. \& Stefani, F. 2016 Subcritical excitation of the currentdriven Tayler instability by super-rotation. Physics of Fluids 28 (1), 014105.

SCHUltZ-Grunow, F. 1959 Zur Stabilität der Couette-Strömung. Zeitschrift für Angewandte Mathematik und Mechanik 39, 101-110.

Seilmayer, M., Galindo, V., Gerbeth, G., Gundrum, T., Stefani, F., Gellert, M., RÜDiger, G., Schultz, M. \& Hollerbach, R. 2014 Experimental Evidence for Nonaxisymmetric Magnetorotational Instability in a Rotating Liquid Metal Exposed to an Azimuthal Magnetic Field. Physical Review Letters 113 (2), 024505.

Seilmayer, M., Stefani, F., Gundrum, T., Weier, T., Gerbeth, G., Gellert, M. \& Rüdiger, G. 2012 Experimental Evidence for a Transient Tayler Instability in a Cylindrical Liquid-Metal Column. Physical Review Letters 108 (24), 244501.

Shalyb Kov, D. 2006 Pinch instabilities in Taylor-Couette flow. Physical Review E 73 (1), 016302.

StefANi, F. \& KiRILlov, O. N. 2015 Destabilization of rotating flows with positive shear by azimuthal magnetic fields. Physical Review E 92 (5), 051001.

TAYLER, R. J. 1957 Hydromagnetic Instabilities of an Ideally Conducting Fluid. Proceedings of the Physical Society B 70, 31-48.

TAYLER, R. J. 1973 The adiabatic stability of stars containing magnetic fields-I.Toroidal fields. Month. Not. Roy. Astr. Soc. 161, 365.

TAYLOR, G. I. 1936 Fluid Friction between Rotating Cylinders. I. Torque Measurements. Proceedings of the Royal Society of London Series A 157, 546-564.

Terquem, C. \& Papaloizou, J. C. B. 1996 On the stability of an accretion disc containing a toroidal magnetic field. Month. Not. Roy. Astr. Soc. 279, 767-784.

VAndakuRov, Y. V. 1972 Theory for the Stability of a Star with a Toroidal Magnetic Field. Soviet Astronomy 16, 265.

VELIKHOV, E. 1959 Stability of an ideally conducting liquid flowing between cylinders rotating in a magnetic field. Soviet. Phys. JETP 36, 1389-1404.

WENDT, F. 1933 Turbulente Strömungen zwischen zwei rotierenden koaxialen Zylindern, Diss. Göttingen 1934. Ingenieur-Archiv 4, 557-595. 